\title{
Décision et incomplétude
}

\section{BOISSIER}

LERMES/CUST

BP 206

63174 Aubière Cedex
La prise de décision en génie civil se fait dans un contexte où imprécis et incertain cohabitent : imprécis des objectifs assignés et de la connaissance du sol, imprécis des modèles utilisés, inadéquation entre la précision des modèles et le niveau de décision, mais aussi incertain des actions qui vont solliciter les ouvrages, incertain du comportement des ouvrages. Dans ce texte, nous montrons tout d'abord l'importance de I'imprécision et de l'incomplétude des informations en géotechnique; nous proposons l'apport de la théorie des sous-ensembles flous (SEF) et des fonctions de croyance dans l'aide à la résolution des problèmes de géotechnique. Nous rappelons tout d'abord les concepts et bases de ces théories (partie A) puis, nous appuyons sur quatre exemples concernant les fondations pour en proposer différentes utilisations (partie B).

Mots clés : aide à la décision, incertain, imprécis, sousensembles flous, géotechnique.

\section{Decision making and incompleteness}

Decision making in civil engineering takes place in an uncertain and imprecise context. The assigned objectives are imprecise such as soil knowledge and bad adapted models. Loads and load effects deal with uncertainty and the behaviour of the works is less or more predictable. In this paper we show that in the geotechnics field data are very imprecise and uncertain ; we propose to introduce the use of the fuzzy sets and of the belief functions theories. We first present the theoretical bases in Part $A$, then four examples in part B. in order to illustrate the use of these concepts in the geotechnics field.

Key words : decision making, incertain, imprecise, fuzzy sets, geotechnics. 


\section{Partie A - CONCEPTS ET MÉTHODES}

\section{Introduction}

"Le bon sol $\%$, " un sol compressible $», ~ «$ une portance d'environ 0,2 Mpa », " une couche homogène », " une cohésion entre 20 et $30 \mathrm{kPa} »$, « un sol saturé $»$, « la fondation est surdimensionnée $»$, a la nappe est variable $). .$. Toutes ces expressions ont en commun deux caractéristiques:

1) elles sont communément utilisées et comprises par des géotechniciens : cis.

2) elles sont toutes à support d'information non pré-

Ce texte montre la nécessité d'approches non traditionnelles pour la prise en compte de ce type d'information par les mécaniciens des sols; il propose quelques repères pour l'aide au choix de modèles possibles de représentation et de traitement. Il s'appuie sur des travaux de recherche que j'ai dirigés à l'université d'Artois (J. Al-Hajiar, H. Mammeri, I. Semaan, A. Karnib), puis à l'université Blaise Pascal (J. Lair) et sur d'autres travaux qui sont en cours sur ce theme au sein de l'équipe Mécanique des sols du Lermes avec C. Bacconnet et R. Gourvès.

\section{2}

\section{Constat d'échec et proposition}

La théorie des ensembles et la théorie des probabilités ne permettent pas toujours de rendre compte d'une réalité complexe. En effet, l'information disponible est souvent imparfaite (incomplète, imprécise, inadaptée, suspecte...) ; elle ne provient pas toujours non plus d'une mesure (dire d'expert, estimation...); enfin, les modèles de l'ingénieur ne permettent pas de modéliser une réalité complexe où les décisions sont prises au regard de considérations diverses.

\section{1}

\section{Le constat de L.A. Zadeh (1965)}

Au fur et à mesure que la complexité des systèmes augmente, notre aptitude à formuler de manière précise et significative leur comportement diminue jusqu'á une limite au-delà de laquelle la précision et la signification deviennent des caractéristiques mutuellement exclusives.

Notre connaissance du monde réel est imprécise et celle de son évolution incertaine; il est vain alors de vouloir le décrire par des modèles précis et déterministes ; cela est vain et surtout dangereux car le sentiment de précision est illusoire, la signification des résultats est très pauvre et les décisions sont prises sur la base d'informations imprécises et sans signification.

Deux arguments sont alors mis en évidence : la fiabilité de l'information et son « informativité $n$; il apparait vite que ces deux arguments sont souvent conflictuels.
- Exemple 1: la cohésion du sol dans cette couche est de $20 \mathrm{kPa}$.

Cette information est très informative et l'on peut faire des calculs ou prendre des décisions sur cette base, par contre elle est très peu fiable; en effet en pratique l'on ne peut pas assurer que le sol a une cohésion de $20 \mathrm{kPa}$; tout au plus peut-on dire que la mesure dans telle condition sur tel appareil de tel échantillon provenant de tel prélèvement a donné une valeur que l'on arrondit à $20 \mathrm{kPa}$.

- Exemple 2: la cohésion du sol dans cette couche est comprise entre $10 \mathrm{kPa}$ et $100 \mathrm{kPa}$.

Cette information est très fiable; il y a très peu de chance que dans cette même couche de sol une autre mesure donne une valeur inférieure à $10 \mathrm{kPa}$ mais par contre elle n'est que très peu informative et it est difficile de faire des calculs ou de prendre des décisions sur cette base ; ce sont des valeurs numériques que l'on introduit dans des codes de calcul, parfois des distributions de probabilités et c'est une valeur que l'on compare à une limite ou un index qui sert à prendre une décision ; que faire alors d'une information sous forme d'un intervalle ou d'un ensemble?

\section{2}

\section{Décision en ingénierie (Boissier D., Al-Hajjar J., 1990)}

\section{1 \\ Décision et précision}

Dans les problèmes d'ingénieur, il est trivial de rappeler que c'est en début de résolution que l'espace des solutions est le plus grand mais que c'est aussi à ce moment-là que l'espace des données disponibles est le plus petit et que les conséquences d'une décision sont les plus importantes car réduisant fortement l'espace des solutions ; à ce moment les données sont souvent de mauvaise qualité et la stabilité de la décision ne peut être assurée (on aurait pris une autre décision si lion avait eu toutes les informations...).

- Exemple: La première reconnaissance visuelle d'un site est un des critères de décision d'achat d'une parcelle en vue d'une opération immobilière ; la connaissance du sol est alors incomplète et de mauvaise qualité ; par contre la décision d'investissement est lourde de conséquences. A l'inverse, en fin de conception, lorsque les études de sol auront été dépouillées, il sera possible « d'optimiser n les fondations et d'économiser quelques $\mathrm{m}^{3}$ de béton ; le gain marginal est faible, le risque aussi car le problème est mieux défini. Il se pourrait aussi qu'avec toutes les informations disponibles à ce moment-là on souhaite revenir sur la décision d'investir alors que cela n'est plus possible ; la décision est donc conditionnée par la qualité de l'information.

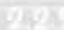

\section{Informations disparates}

Les informations disponibles pour résoudre un problèmé donné sont souvent disparates : elles proviennent de sources diverses (observations, mesures 
diverses, bibliographie, dire d'experts) et sont exprimées sous formes différentes.

Elles peuvent être incohérentes, incompatibles, redondantes et plus ou moins fiables (la fiabilité est liée à la valeur associée à l'information et non à la variabilité intrinsèque de l'information). Pourtant, pour prendre une décision, il faudra agréger toutes ces informations et souvent les ramener à une information unique.

- Exemple: On dispose d'une stratigraphie donnée par le géoloque et d'un découpage des couches selon leur résistance mécanique obtenue à partir de l'analyse d'un signal pénétrométrique. Comment choisir la profondeur d'ancrage de fondations?

\section{3}

\section{Un constat positif}

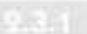

\section{Perception globale et langage}

L'être humain possède des capacités de perception globale des systèmes complexes. Il est souvent capable de porter " intuitivement " ou par " expertise non explicite » un jugement plus sûr, c'est-à-dire plus stable en décision, que celui obtenu par des modèles précis mais trop restrictifs (hypothèses non justifiables ou non crédibles).

L'être humain est aussi capable d'exprimer le vague et l'imprécis par le langage; or ce langage est éloigné de celui utilisé dans les théories traditionnelles des ensembles et des probabilités.

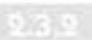

\section{Quelques exemples en ingénierie}

a) la visualisation d'un système constructif permet au concepteur de détecter une erreur de conception (appui sur ou sous abondant, réservation trop petite, croisement de réseaux impossible, superposition de structures incorrecte) alors que le calcul géométrique ou mécanique dans un système de CAO ne le permet pas;

b) un ingénieur peut pour un problème donné caractériser un sol de " mauvais », alors qu'aucun modèle mécanique ne permettrait de calculer sa résistance (hétérogénéitê, présence de cavités...) ;

c) on peut affirmer que la charge d'une structure sera d'environ $x$. L'égalité n'a pas de sens car au moment du calcul la structure n'existe pas encore et parce qu'au cours de sa vie la charge peut varier; ce qualificatif environ est peu précis et on ne sait exactement ce qu'il recouvre mais il montre explicitement que l'on est dans une ignorance relative :

d) on peut affirmer qu'un sol est compressible ; ce qualificatif le sépare de la catégorie des sols incompressibles. La limite « compressible/incompressible » n'est pas nette; elle est relative à des indicateurs (l'indice de compression est un indicateur parmi d'autres) : pour un matériau donné, la valeur obtenue pour l'indicateur est liée à la précision de la mesure (technique de mesure, conditions opératoires) et à l'échantillon (variabilité spatiale et temporelle) :

e) les règles de décision sont souvent basées sur des classifications (sable/gravier, sec/humide) qui en réalité ont des domaines d'intersection non vides (un même sol peut ne pas appartenir à la même catégorie dans des classifications différentes).

\section{3}

\section{Apport de la logique floue}

\section{1}

\section{Définitions}

Imprécis : Il concerne des informations mal définies; on lui associe les qualificatifs de type : " environ », "à peu prés », " dans l'intervalle $»$, " compris entre et entre $w$, "approximativement ${ }^{\prime} . .$. ; il représente la qualité de la connaissance que nous avons d'un phénomène.

Incertain : Il résulte essentiellement d'une êpreuve dont le résultat n'est pas connu a priori et peut changer si on la recommence; il représente la variabilité d'un phénomène; il peut être représenté de façon rigoureuse et précise.

Dans notre domaine, certaines données (charges et résistances par exemple) sont, par nature même, incertaines; leur variabilité spatio-temporelle est irréductible (données climatiques, propriétés des géomatériaux et des matériaux de construction, charges d'exploitation...).

Remarque : Il ne faut pas confondre la qualité d'une donnée et la qualité de l'information que l'on possède sur la donnée.

\section{La théorie des sous ensembles flous (Fuzzy sets)}

\section{$36 a_{1}$}

\section{Présentation (Bouchon Meunier, 1995)}

L'idée de base est que lorsqu'une classe est définie par une notion vague, on ne peut pas utiliser le concept classique d'appartenance ; il faut alors introduire une échelle - la fonction d'appartenance - qui permette de hiérarchiser les éléments d'un univers donné par rapport à cette propriété vague.

Ici c'est l'ensemble qui est flou (ou du moins ses limites) par opposition à un ensemble ( $\propto$ net $»)$ traditionnel dont les limites sont précises; la fonction d'appartenance exprime si une valeur $\mathrm{x}$ précise satisfait pleinement ou non les propriétés de l'ensemble.

L'ensemble classique (ou net) est un cas particulier où l'appartenance est binaire : $x$ appartient ou n'appartient pas à l'ensemble. En théorie des possibilités, c'est l'ensemble qui est net alors que la mesure (donnée) est floue.

\section{$\sin 2$}

\section{Définition}

La théorie des sous-ensembles flous (SEF) a été développée par L.A. Zadeh (1965); elle permet de 
modẻliser l'imprécis et l'incertain d'une autre manière que par les modèles stochastiques. Les SEF sont une voie d'introduction de l'incertain et de l'imprécis dans l'aide à la décision.

Soit $X$, un ensemble de référence; un sousensemble flou A de X peut être défini à partir de la fonction d'appartenance $\mu_{\mathrm{A}}$ telle que :

$$
\forall x \in X-\cdots \mu_{A}(x) \in[0,1]
$$

L'application $\mu_{A}$ (éq. 1) est appelée fonction d'appartenance et la valeur $\mu_{A}(\mathrm{x})$ est appelée niveau d'appartenance.

\section{$3.9 \times 5$}

\section{Propriétés}

Le support de A, noté $\operatorname{supp}(\mathrm{A})$ est la partie de X pour laquelle $\mu_{A}(x)>0$.

La hauteur, notée h(A) est la plus grande valeur de la fonction d'appartenance. $h(A)=\sup _{x \in X}\left(\mu_{A}(x)\right)$

Le noyau, noté noyau(A), est l'ensemble des valeurs de A pour lesquelles la fonction d'appartenance est maximale.

Lorsque l'ensemble X est à valeurs discrètes, la cardinalité est la somme des valeurs des fonctions d'appartenance.

\section{4}

\section{Cas particulier}

Il existe de nombreuses variétés de sous-ensembles flous ; nous nous limitons ici aux SEF de type L_R normés; leur fonction d'appartenance est paramétrée par deux fonctions $L$ et $R$ et quatre paramètres $m, n, \alpha, \beta$ et leur hauteur est égale à 1 ; nous pouvons voir un SEF de type L_R trapèze noté $A(m ; n ; \alpha ; \beta ; 1)$ (Fig. 1a) et L_R triangle symétrique noté $A(m ; \alpha ; \beta ; 1)$ (Fig. 1b).

Contrairement aux ensembles ordinaires, un SEF n'a pas une appartenance nettement définie mais comporte des transitions graduelles d'appartenance depuis l'appartenance totale (degré 1) jusqu'à l'absence d'appartenance (degré 0).

Ce concept peut être illustré comme suit : quand le niveau d'appartenance est près de 1 alors nous pou- vons assurer que la proposition « $\mathrm{x}$ appartient à $\mathrm{F} »$ est satisfaite ; lorsque ce niveau est près de 0 la proposition n'est pas satisfaite; la validité de la proposition peut donc être liée à la valeur $\mu_{-}(\mathrm{x})$. L'exemple 1 illustre l'utilisation de ce concept en fiabilité.

\section{2 .5}

\section{Compatibilité entre deux sous-ensembles flous}

Soit $X$ un ensemble de référence, A et B 2 SEF et $\mu_{A}(x)$ et $\mu_{B}(x)$ leurs fonctions d'appartenance respectives; soit $\mu_{\mathrm{A}}^{-1}(\mathrm{x})$, la fonction inverse de $\mu_{A}(\mathrm{x})$. Alors, Zadeh L.A. (1978) et Dubois D. et Prade H. (1983) définissent la compatibilité entre A et B sous la forme d'une distribution des valeurs possibles du degré de compatibilité ; celle-ci est alors appelée profil de compatibilité ; elle est considérée comme un SEF C, dont la fonction d'appartenance $\mu_{c}$ (x) est calculée par l'équation 2 et qui est définie sur lé domaine $\mathrm{X}_{c}=[0,1]$.

$\mu_{c}(\mathrm{t})=\sup \left(\mu_{\mathrm{A}}(\mathrm{x})\right.$ avec $\mathrm{x} \in \mathrm{X}$ et tel que $\mu_{B}(\mathrm{x})=\mathrm{t}$ $\mu_{c}(t)=0$ si $\mu_{B-1}(t)=0$

Pour toute valeur t du degré de compatibilité, la procédure de calcul est la suivante:

1) On recherche toutes les valeurs de B qui ont un degré d'appartenance t ;

2) On calcule le degré d'appartenance de chacune de ces valeurs au SEF A ;

3) Le maximum de ces degrés d'appartenance est le degré d'appartenance du degré de compatibilité t.

Si le degré d'appartenance t ne correspond à aucune des valeurs du SEF B, alors le degré de compatibilité de t est nul.

Cette procédure est illustrée sur la figure 2 et utilisée dans l'exemple 2.

\section{$\operatorname{sic} 3$}

\section{Comparaison de deux sous-ensembles flous}

Soient A et B, deux sous-ensembles flous ; l'objectif de cette comparaison est de voir dans quelle mesure une observation A peut ètre considérée comme une observation de l'ensemble B de référence; lorsque A

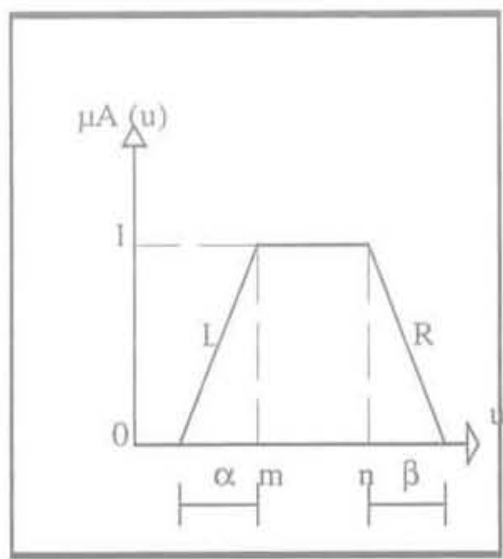

FIG 1A SEF L R trapèze. Trapezium L_R fuzzy Set.

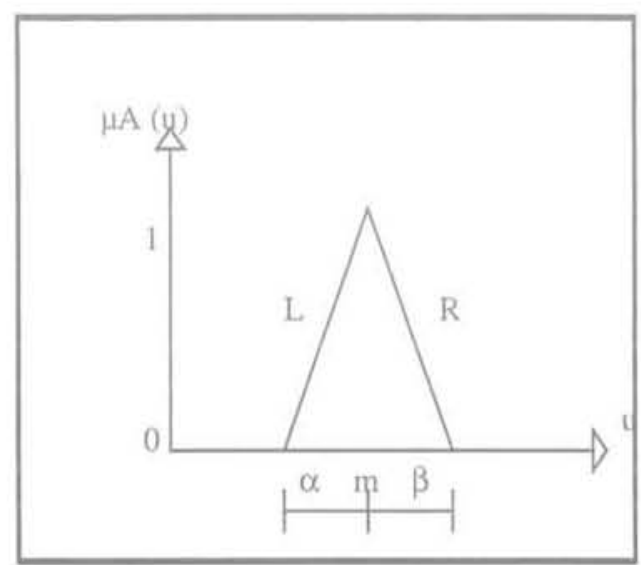

Fig. 1b SEF L R triangle. Triangle L_R fuzzy Set. 

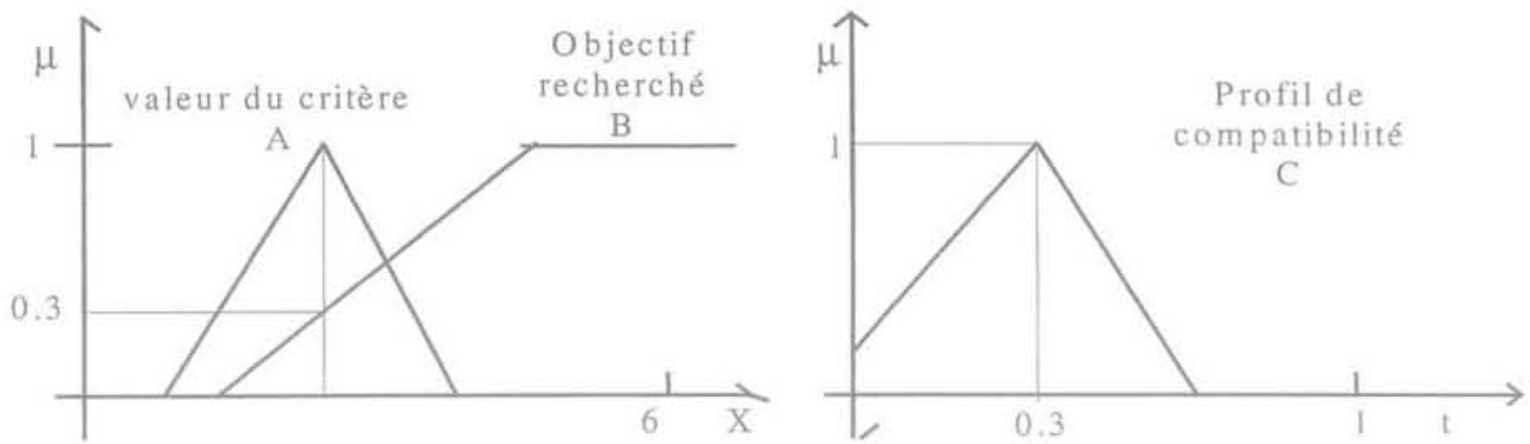

FiG. \& Profil de compatibilité entre deux sous ensembles flous.

Profile of compatibility between two fuzzy sets.

et B sont deux SEF, il s'agit de comparer des intervalles auxquels sont associées des fonctions d'appartenance. La méthode de comparaison des intervalles flous est basée sur les mesures de cardinalités floues; $|\mathrm{A}|$ la cardinalité floue de A est définie par l'équation 3 :

$$
|\mathrm{A}|=\sum_{\mathrm{X} \in \mathrm{X}} \mu_{\mathrm{A}}(\mathrm{X})
$$

$\mathrm{Cp}(\mathrm{A} / \mathrm{B})$, la cardinalité floue relative, est un degrè de vraisemblance qui traduit combien A peut être considérée comme une observation de $\mathrm{B}$ par le biais de la proportion de paramètres satisfaits dans $A$, compte tenu de B.

N. Aggaggarwal, P. Quarrey, C. Berry (1993) calculent $\mathrm{Cp}$ par la règle du tableau I:

Pour des SEF continus, le calcul du degré de vraisemblance par la cardinalité floue revient au calcul de l'aire de l'intersection des deux intervalles. Si cette intersection est vide alors la cardinalité relative est nulle.

Cette formulation à deux SEF peut être étendue au cas où l'on veut comparer le SEF A à plusieurs SEF de type $B$; un algorithme de discrimination permet alors de trouver quel est l'ensemble B qui est le plus proche du SEF A cou réciproquement à quel SEF B peut être rattaché avec le plus de vraisemblance le SEF A). L'exemple 3 illustre l'utilisation de cette méthode.

\section{3}

\section{La théorie des fonctions de croyance (Dempster Shafer)}

\section{3is.t \\ Présentation}

Cette théorie, dont les bases ont été établies en 1976 par G. Shafer (1976), permet de représenter l'ignorance et l'incertain, de prendre en compte toutes les informations existantes sur un sujet donné quels que soient leur provenance, leur format, et de dégager une information consensuelle. Il a proposé des règles de « fusion des données » ou “ d'unification de données » quỉ sont basées sur les hypothèses suivantes :

- de toute information il y a quelque chose à déduire; - à chaque information est attachée une masse de croyance, c'est-à-dire simplement un attribut précisant la qualité de cette information.

Le problème alors est de construire (ou de calculer) une information résultante à partir de plusieurs informations; la méthode est basée sur l'idée qu'il faut prendre en compte en même temps l'information (ou sa modélisation) et sa qualité (ici la masse de croyance); c'est ce que le décideur fait, de manière non formelle souvent, lorsque après avoir écouté les experts il se

TABIEAU Calcul du degré de vraisemblance $C p(A / B)$. Calculus of the $\mathrm{Cp}(\mathrm{A} / \mathrm{B})$ likelihood degree.

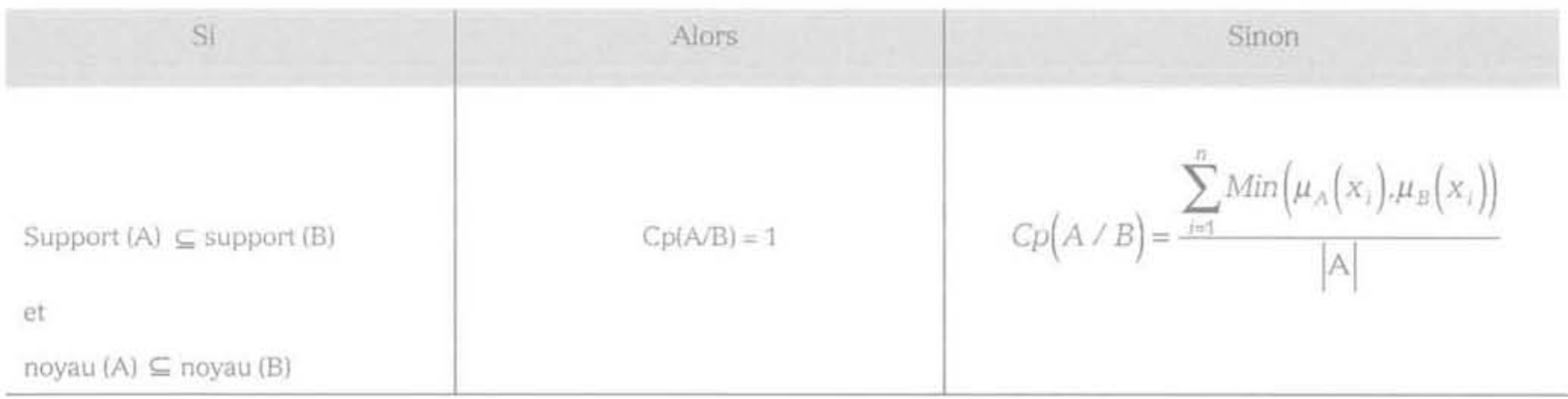


"fait une opinion $)$ et décide. Il prend en compte l'information (donnée, assertion...) mais aussi sa provenance sachant que certaines sources sont sûres alors que d'autres sont moins fiables, que certains modèles ou analogies sont près de la réalité, d'autres plus éloignés.

Une difficulté est que parfois les informations sont contradictoires et donc que des dires d'experts ou des résultats expérimentaux sont infirmés. L'information résultant de deux expériences en partie ou complètement contradictoires a cependant une signification et une qualité qu'il faut déterminer.

\section{$\exists=12$}

\section{Principe}

Supposons un ensemble $\Theta$ appelé u cadre de discernement n correspondant à l'ensemble des réponses à une question donnée (par exemple si la question est "quelle est la cohésion du sol ? p, alors $\Theta$ est l'ensemble des cohésions : $\Theta=[0, \mathrm{c}]$. Ce cadre de discernement doit être exhaustif, ce qui signifie que la solution (ici la cohésion) appartient obligatoirement au cadre de discernement. Toute réponse à la question est appelée une preuve et pointe sur un sous-ensemble $S$ inclus dans $\Theta$ ; les sous-ensembles pointés peuvent être une mesure ponctuelle, un intervalle, une densité de probabilité, un ensemble flou...

Toute preuve est obtenue dans un « contexte d'acquisition $x$ particulier et c'est à partir de l'analyse de ce contexte d'acquisition que l'on peut apprécier la qualité de la preuve S. Dans la théorie de Shaffer, la "qualité d'une preuve » est représentée par une quantité $m(S)$ associée à chacun des sous-ensembles; c'est un nombre compris entre 0 et 1 qui représente la force de la preuve, sa « fiabilité 刃. L'estimation de ces masses peut se faire subjectivement en mode déclaratif ou être guidée par une grille d'analyse.

En réponse à une question telle que « quelle est la cohésion du sol n, des exemples de preuve sont la réponse donnée par un expert qui annonce une cohésion entre 20 et $40 \mathrm{kPa}$, le résultat d'un essai in situ grâce auquel on estime une cohésion de $40 \mathrm{kPa}$, une étude des dossiers sur des bâtiments existants qui montre que les calculs ont été faits avec une cohésion moyenne de $35 \mathrm{kPa}$, etc, ; toutes les sources dont sont issues ces données ont des "fiabilités différentes ".

Ayant toutes les informations sous forme de couples [S: information ; $\mathrm{m}$ : croyance], nous pouvons les fusionner au moyen de la règle de Dempster Shaffer (\$333), afin d'obtenir un consensus.

\section{3is:3}

\section{Unification de deux informations (J. Lair, 2000)}

Soit deux preuves $\mathrm{P}_{2}$ et $\mathrm{P}_{2}$ pointant respectivement sur $A$ et $B$, de masses de croyance $m_{a}$ et $m_{b}$; distinguons les deux situations suivantes : $A \cap B \pi \varnothing$ et $A \cap$ $\mathrm{B}=\varnothing$.

\section{- Preuves concordantes $(A \cap B \pi \varnothing)$}

Pour modéliser la connaissance contenue dans la preuve pointant sur $A$ (soit $P_{1}$ ), nous affectons une masse ma sur le sous-ensemble $A$, une masse $\left(1-m_{a}\right)$ sur $\bar{A} \cap B$ et $\left(1-m_{n}\right)$ sur l'ignorance $\overline{A \cup B}$. L'ignorance est en fait une indécision : on affecte la masse $\left(1-m_{a}\right)$ sur $\Theta$ puisque l'on ne sait pas.

Par analogie, pour modéliser la connaissance contenue dans la preuve pointant sur $\mathrm{B}\left(\mathrm{P}_{2}\right)$, nous affectons une masse $m_{b}$ sur $B$, une masse $\left(1-m_{b}\right)$ sur $A \cap \bar{B}$ et $\left(1-m_{b}\right)$ sur l'ignorance $\overline{A \cup B}$ (Tableau II)

Le consensus est obtenu en affectant des masses aux sous-ensembles comme ci-après :

pour $A \cap \bar{B}$ : ma. $\left(1-m_{b}\right)$, pour $A \cap B$ : ma.mb, pour

$B \cap \bar{A}: m b \cdot(1-m)$ et pour $\overline{A \cup B}:\left(1-m_{a}\right) \cdot\left(1-m_{b}\right) . L a$ somme des quatre masses consensuelles donne 1. Le produit $\left(1-m_{a}\right) \cdot\left(1-m_{b}\right)$ correspond à l'ignorance et reflète le cas où les deux preuves seraient fausses.

\section{- Preuves conflictuelles $(A B=\varnothing)$ (Tableau III) \\ De la même manière :}

- pour modéliser la connaissance contenue dans la preuve pointant sur $\mathrm{A}$, nous affectons une masse ma sur le sous-ensemble $A$, une masse $\left(1-m_{n}\right)$ sur $B$ et $\left(1-m_{u}\right)$ sur l'ignorance ;

- pour modéliser la connaissance contenue dans la preuve pointant sur $B$, nous affectons une masse mb sur le sous-ensemble $B$, une masse $\left(1-m_{b}\right)$ sur $A$ et $\left(1-m_{b}\right)$ sur l'ignorance.

De nouveau, le consensus est obtenu en affectant le produit des masses aux sous-ensembles concernés. La somme des quatre masses consensuelles donne $\left(1-m_{a} m_{b}\right)$.

Cet écart $m_{0} \cdot m_{b}$ caractérise le caractère conflictuel des données, c'est-à-dire la masse que l'on affecte à un ensemble inexistant. La règle de Dempster nous dit de répartir la masse conflictuelle proportionnellement sur les trois autres masses en appliquant un facteur de normalisation $\mathrm{k}$ (eq. 4).

$k=\frac{1}{1-m_{c}}$ où $m_{c}$ est la masse affectée à l'ensemble vide

Soit deux preuves $\mathrm{P}_{3}$ et $\mathrm{P}_{2}$ pointant respectivement sur les sous-ensembles $A$ et $B$, de masses de croyance $m_{3}(A)$ et $m_{b}(B)$, la règle d'assemblage de Dempster, donnant la répartition des masses résultantes est (ec. 5):

$$
m(\theta)=k \sum_{\substack{A_{j} \cap B_{j=\theta} \\ m_{j}}} m_{n}\left(A_{i}\right) \cdot m_{b}\left(B_{j}\right)
$$

L'application de Dempster dans le cas de deux données conflictuelles aboutit donc à l'équation 6 :

$$
\mathrm{m}(\mathrm{A})=\frac{1}{1-\mathrm{m}_{\mathrm{a}} \cdot \mathrm{m}_{\mathrm{b}}} \cdot \mathrm{m}_{\mathrm{H}} \cdot\left(1-\mathrm{m}_{\mathrm{b}}\right)
$$

Nous obtenons ainsi une nouvelle répartition des masses. La " preuve résultante » pointe maintenant sur A, sur B, sur l'ignorance et sur $A \cap B$ (cas de preuves concordantes). C'est une preuve fictive qui englobe toute la connaissance que nous avions dans les preuves initiales. Le principe de la règle de Dempster est donc que deux éléments concordants se renforcent mutuellement et deux éléments discordants s'érodent. 


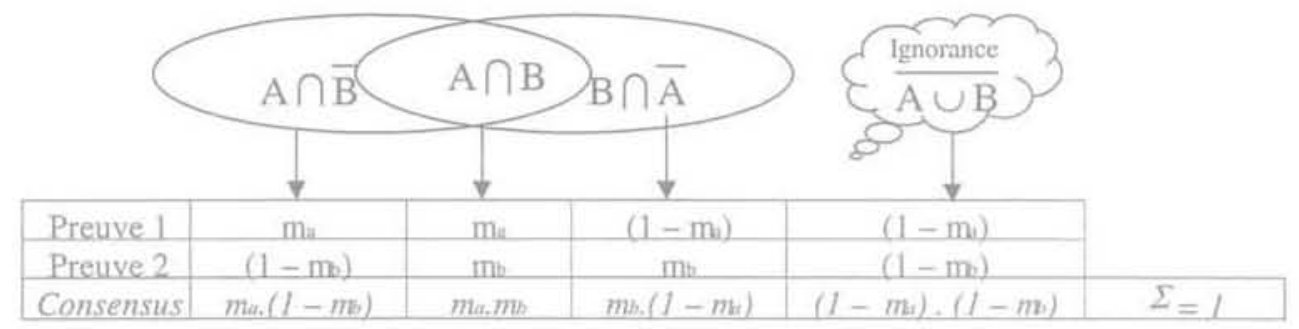

TABLEAU III Affectation des masses. Cas des preuves conflictuelles. Masses affectation for conflicting evidences.

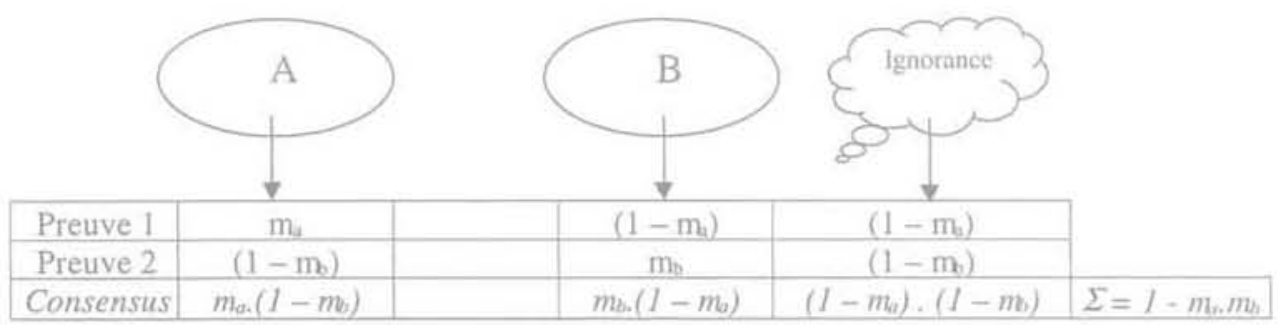

En utilisant ces règles d'assemblage, nous fusionnons deux à deux toutes les preuves disponibles. Nous obtenons ainsi une répartition de masses sur les sousensembles de $\Theta$ pointés par les preuves et sur ceux générés par le processus de fusion (intersections). L'exemple 4 illustre ce concept.

\section{Partie B - EXEMPLES EN GEOTECHNIQUE}

\section{4}

\section{Exemple 1 : optimisation d'une fondation selon sa fiabilité}

Sur ce premier exemple nous utilisons le concept de fiabilité possibiliste basé sur l'utilisation de SEF et introduit par Boissier D., Alhajiar J., Mammeri H. (1995).

\section{1}

\section{Définitions}

\section{- Fiabilité}

La fiabilité d'une structure est son aptitude à remplir ses objectifs de conception pendant une durée de référence spécifiée; la défaillance d'une structure est son incapacité à remplir ses objectifs.

\section{- Fiabilité probabiliste}

En contexte probabiliste, la fiabilité est liée à la probabilité de défaillance par référence à un état limite. Les méthodes fiabilistes prennent en compte l'aléa de chaque information initiale à laquelle le décideur peut associer une modélisation stochastique ; il introduit alors ces modélisations dans des codes probabilistes qui évaluent une probabilité de défaillance $\left(\mathrm{P}_{\text {t }}\right)$ ou un index de fiabilité tel que l'index $\beta_{H}$ proposé par Hasofer A.M., Lind N.C. (1974).

\section{- Fiabilité possibiliste}

Dans un contexte incertain et imprécis nous définissons la fiabilité possibiliste comme la compatibilité entre une proposition de solutions et des états limites (objectifs).

L'analyse possibiliste consiste alors à associer à cette compatibilité un degré de vraisemblance. Elle prend en compte l'imprécis des informations à laquelle le décideur associe un modèle possibiliste, en général un sous-ensemble flou (SEF) ; un code de calcul possibiliste permet alors d'associer une vraisemblance aux résultats à comparer aux objectifs.

\section{2}

\section{Cas du dimensionnement d'une fondation}

\section{Cas d'étude et fiabilité déterministe}

L'exemple est celui du calcul de la portance ultime $\mathrm{q}_{u}$ d'un élément de fondation superficielle à partir des résultats d'essais de laboratoire. Nous considérons une semelle filante soumise à une charge verticale et uniformément répartie sur sa longueur. Les hypothèses de l'application sont : théorie de plasticité parfaite, fondations parfaitement rigides, actions verticales et centrées et cas de sol naturel et homogène. 
La portance ultime q (éq. 7) du facteur de sécurité F (éq. 8) et de la probabilité de défaillance $\mathrm{P}_{\mathrm{f}}$ s'exprime par :

$$
\begin{aligned}
& \mathrm{q}_{\mathrm{u}}=\frac{1}{2} \gamma^{*} \mathrm{~B} * \mathrm{~N}_{\gamma}+\mathrm{c}^{*} \mathrm{~N}_{\mathrm{c}}+\gamma^{*} \mathrm{D} \mathrm{N}_{\mathrm{q}} \\
& \mathrm{F}=\mathrm{q}_{\mathrm{u}} / \mathrm{q} \\
& \mathrm{Pf}=\text { Probabilité }\left(\mathrm{q}_{\mathrm{u}} / \mathrm{q}<1\right)
\end{aligned}
$$

avec $B$ : largeur (ici $B=1,50 \mathrm{~m}$ ) ; $\mathrm{D}$ : profondeur (ici $\mathrm{D}=$ $0,50 \mathrm{~m})$; $\mathrm{C}$ : cohésion: $\mathrm{n}$ : angle de frottement interne ; $\varphi$; poids volumique des terres; $q$ : charge; $N_{1} N, N_{q}$; facteurs de surface, de cohésion et de profondeur.

Les causes d'incertain sont la variation spatio-temporelle des propriétés du sol et de la charge appliquée; les causes d'imprécision sont l'empirisme et la simplification des modèles mécaniques et des méthodes de calcul, la mauvaise qualité de la reconnaissance (incomplétude, imprécision des mesures, irrégularité des sondages....).

\section{9}

\section{Fiabilité probabiliste}

Les espérances mathématiques des données sont résumées dans le tableau IV (CV est le coefficient de variation) ; les variables aléatoires sont supposées gaussiennes et indépendantes.

\section{TABEEAUIV Données probabiliste.} Data: laws of probability.

\begin{tabular}{l|c|c|c|c|c|c|c|}
\hline$q(\mathrm{kN} / \mathrm{m})$ & $\mathrm{CV}$ & $\mathrm{C}(\mathrm{kPP})$ & $\mathrm{CV}$ & $\varphi \mathrm{d}^{\circ}$ & $\mathrm{CV}$ & $\gamma\left(\mathrm{kN} / \mathrm{m}^{3}\right)$ & $\mathrm{CV}$ \\
\hline 200 & 0,10 & 30 & 0,20 & 20 & 0,10 & 20 & 0,05 \\
\hline
\end{tabular}

Le calcul probabiliste du critère $F=q_{d} / q$ (éq. 8) donne une probabilité de défaillance $\mathrm{P}_{f}=\operatorname{Proba}[\mathrm{F}<1]=$ $3,90.10^{-3}$ (éq. 9) et un indice de Lind Hasofer $\beta_{\mathrm{HL}}=$ $-2,66$.

Deux solutions ou deux variantes de dimensionnement peuvent alors être comparées à partir de ces critères; l'interprétation est simple mais les approximations, estimations et hypothèses inhérentes à cette méthode ne sont pas explicites.

\section{Acos?}

\section{Fiabilité possibiliste}

Nous décrivons la connaissance que nous avons de chaque caractéristique par un intervalle de valeurs numériques auquel nous associons un sous-ensemble flou : ce modèle quantitatif peut être alors introduit dans un code de calcul. Le degré de vraisemblance associé à chacune des valeurs, bien qu'il soit subjectif, donne une information sur la qualité de la caractéristique ; il provient du jugement que nous pouvons porter sur la représentativité de la caractéristique analysée dans le massif (par référence à des caractéristiques bibliographiques, en fonction de la qualité de la mesure...) ou de l'action.
Ici, nous avons associé un SEF de type trapézoïdal à chaque donnée (tableau V).

La portance ultime qu et le facteur de sécurité F sont calculés à partir des équations (7) et (8) par les opérations de convolution sur les SEF associés à $C, \varphi$ et $\gamma$; l'imprécision de l'angle de frottement $\varphi$ induit des imprécisions sur les facteurs de portance $N_{c} N_{q}$ et $N_{n}$.

TABLEAUV Données possibilistes.

Data : fuzzy sets.

\begin{tabular}{l|c|c|c|c}
\hline Paramètre & $\begin{array}{c}\text { Valeur } \\
\text { inférieure }\end{array}$ & $\begin{array}{c}\text { Valeur } \\
\text { supérietire }\end{array}$ & $\begin{array}{c}\text { Etalement } \\
\text { à gauche }\end{array}$ & $\begin{array}{c}\text { Etalement } \\
\text { a droite }\end{array}$ \\
\hline$C(\mathrm{kPa})$ & 20 & 35 & 5 & 5 \\
\hline$\varphi\left(\mathrm{d}^{\circ}\right)$ & 15 & 25 & 2 & 1 \\
\hline$\gamma\left(\mathrm{kN} / \mathrm{m}^{3}\right)$ & 18 & 22 & 1 & 2 \\
\hline $\mathrm{q}(\mathrm{kN} / \mathrm{m})$ & 160 & 260 & 10 & 20 \\
\hline
\end{tabular}

Le tableau VI récapitule les résultats possibilistes.

TABLEAUVI Résultats possibles. Fuzzy results.

\begin{tabular}{l|c|c|c|c}
\hline Critêre & $\begin{array}{c}\text { Valeur } \\
\text { inférieure }\end{array}$ & $\begin{array}{c}\text { Valeur } \\
\text { supérieure }\end{array}$ & $\begin{array}{c}\text { Étalement } \\
\text { à gauche }\end{array}$ & $\begin{array}{c}\text { Étalement } \\
\text { à droite }\end{array}$ \\
\hline $\mathrm{Q}_{u}(\mathrm{kN} / \mathrm{m})$ & 303,39 & 1049,48 & 92,74 & 266,88 \\
\hline $\mathrm{F}=\mathrm{q}_{\mathrm{u}} / \mathrm{q}$ & 1,2 & 6,5 & 0,5 & 2,2 \\
\hline
\end{tabular}

L'évaluation possibiliste fournit une distribution de possibilité du coefficient $\mathrm{q} / \mathrm{q}$. Cette distribution est caractérisée par un noyau et un support de valeurs possibles. L'objectif est représenté par une valeur ( 3 dans cet exemple, tableau VI et figure 3).

La figure 3 montre qu'il n'y a pas de conflit apparent entre la solution et l'objectif parce que la valeur de l'objectif-3 ici - appartient au noyau de q./q. Si l'objectif avait été de 7 par exemple, la solution n'aurait eu

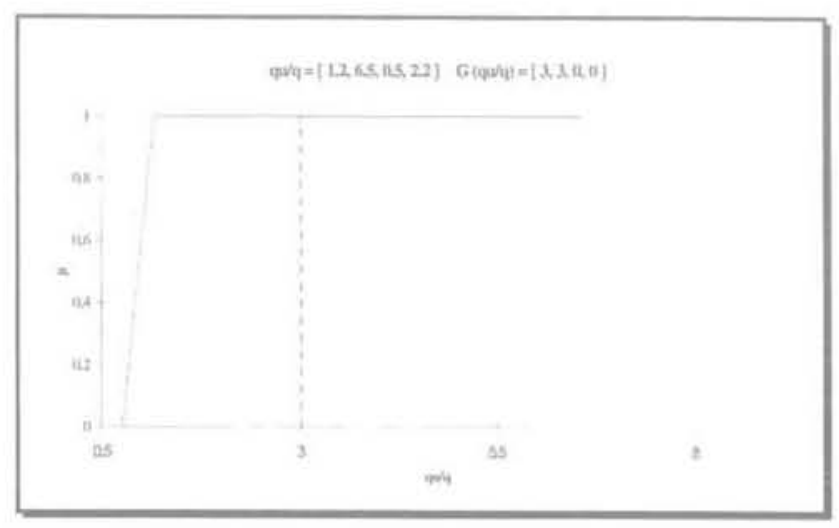

FIG. 3 Comparaison de l'évaluation imprécise et de l'objectif déterministe.

Unprecise assessment and deterministic objective comparison. 
qu'un degré de 0,78 de compatibilité avec lobjectif. Pour un objectif supérieur à 8,8 (sur dimensionnement) ou inférieur à 0,5 (sous dimensionnement) la solution aurait été totalement incompatible.

\section{3}

\section{Conclusion sur l'exemple 1}

L'application au cas de la fondation des deux approches possibilistes et probabilistes pour l'analyse de la fiabilité montre qu'elles sont complémentaires.

L'approche possibiliste nécessite peu d'informations; la modélisation est plus proche des pratiques des concepteurs ; elle est fiable mais peu informative ici.

L'approche probabiliste nécessite des informations beaucoup plus précises ; le langage de modélisation est éloigné des pratiques des concepteurs et cette approche est complexe à mettre en ceuvre : par contre elle se ramène à un critère facilement utilisable dont la précision explicite cache le fait que les lois de probabilités retenues ne sont en rien justifiées.

\section{5}

\section{Exemple 2 : application au tassement d'une fondation superficielle}

Ce deuxième exemple présenté par D. Boissier, J. Alhajjar et H. Mammeri (1995) porte sur l'aptitude d'une proposition technique (ici une solution par semelle filante) à respecter un cahier des charges (ici minimiser le tassement). L'évaluation du tassement, obtenue à partir de mesures, est imprécise, et le tassement limite que l'on ne souhaite pas dépasser est lui aussi imprécis.

II faut alors voir quelle est la compatibilité entre une solution proposée et un objectif à atteindre tous deux imprécis. Une réponse est alors possible sous la forme de la compatibilité entre deux SEF; ici il s'agit de la compatibilité entre le tassement prévisionnel (SEF A) et le tassement admissible (SEF B).

\section{1}

\section{Tassements prévisibles et souhaités}

\subsection{1.}

\section{Tassements prévisibles}

Il s'agit de l'estimation du tassement d'une semelle filante par la méthode pressiométrique de Ménard. Le tassement pressiométrique total $\left(\mathrm{s}_{1}\right)$ s'exprime dans l'équation 11 par la somme de deux termes : le tassement de consolidation $\left(\mathrm{s}_{\mathrm{c}}\right)$ et déviatorique $\left(\mathrm{s}_{\mathrm{d}}\right)$ (éq. 10) :

$$
\begin{aligned}
s_{c} & =\frac{\alpha}{9 E_{M c}}\left(q-q_{0}\right) \lambda_{c} B, s_{d} \\
& =\frac{2}{9 E_{M d}}\left(q-q_{0}\right) \lambda_{d} B_{0}\left(\lambda_{d} \frac{B}{B_{0}}\right)^{\alpha}
\end{aligned}
$$

et

$$
\mathrm{S}_{\mathrm{r}}=\mathrm{S}_{\mathrm{c}}+\mathrm{S}_{\mathrm{d}}
$$

avec $B$ : largeur $(B=1,50 \mathrm{~m}), D$ : profondeur d'ancrage $(\mathrm{D}=1,0 \mathrm{~m}), \mathrm{ql}$ : charge $(\mathrm{ql}=1800 \mathrm{kN} / \mathrm{ml})$ qui donne une contrainte $q$ (ici $q=1,2 \mathrm{MPa}$ ).

A partir d'une analyse géostatistique du profil du module pressiométrique, nous associons à $E_{M c}$ et $E_{M c i}$ deux intervalles flous de représentation trapezoidale (Fig. 1a et éq. 12)

$\mathrm{E}_{\mathrm{Mc}}:(18 ; 20 ; 2 ; 2 ; 1)$ et $\mathrm{E}_{\mathrm{Md}}:(20 ; 25 ; 6,5 ; 28 ; 1)$

Les autres paramètres sont déterministes. Les deux termes du tassement $\mathrm{sc}$ et sd sont obtenus grâce à l'équation 10 ; le tassement total (s.) calculé à partir de l'équation 11 est évalué après linéarisation des fonctions L et R (éc. 13), (Fig. 4) ; nous obtenons:

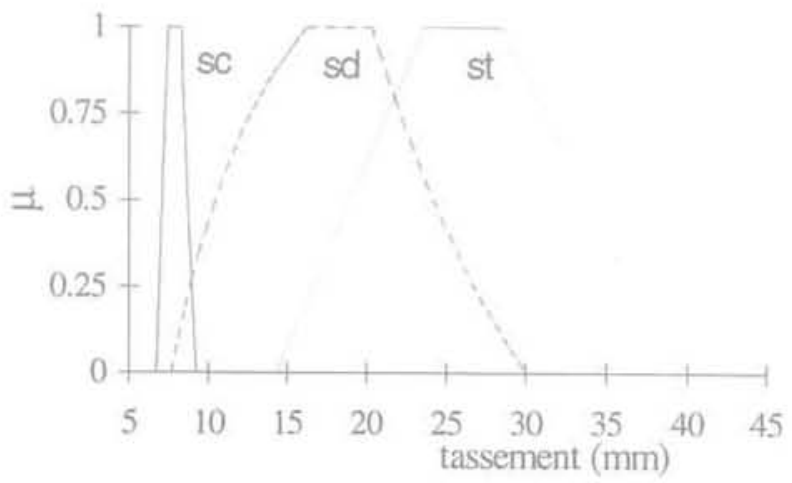

FIG.4 Intervalles flous associés aux tassements. Fuzzy sets for settlement.

$$
\begin{aligned}
& s_{c}:(7,39 ; 8,21 ; 0,67 ; 1,06 ; 1) \\
& s_{d}:(16,23 ; 20,28 ; 8,57 ; 9,77 ; 1) \\
& s_{c t}:(23,61 ; 28,49 ; 9,25 ; 10,83 ; 1)
\end{aligned}
$$

\section{Salla}

\section{Tassements préconisés}

L'objectif associé au tassement (s, est défini par le sous-ensemble flou B (Fig. 5). La déclaration de cet objectif signifie que pour un tassement $<20 \mathrm{~mm}$, la solution est parfaitement compatible $(\mu=1)$, que pour un tassement $>50 \mathrm{~mm}$ la solution est incompatible. $(\mu=0)$ et que pour un tassement $\in[20 \mathrm{~mm}, 50 \mathrm{~mm}]$ l'adéquation de la solution est donnée par un degré de compatibilité $\mu \in[0,1]$.

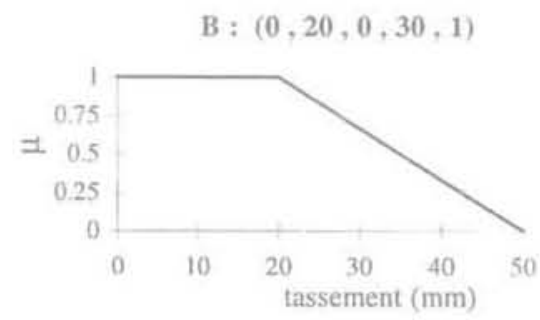

FiG.5 Représentation de l'objectif lié au tassement.

Fuzzy set for objective of settlement. 


\section{Adéquation de la semelle à atteindre l'objectif}

L'adéquation d'une solution à un objectif (fiabilité) est basée sur l'intersection logique des deux intervalles ; la position des noyaux respectifs (Fig. 6) montre l'existence d'un conflit; la nature du conflit est déterminée par la comparaison des positions des supports respectifs (Al-Hajjar J., Boissier D., Boulemia J., 1991).

Les différents degrés de compatibilité sont calculés à partir de l'expression (1) : $\mathrm{A}=$ tassement (s) $)$ : $\mathrm{B}=$ objectif associé. Le profil de compatibilité entre l'objectif et st est représenté par la figure 7. La solution n'est que partiellement compatible avec l'objectif; le degré de compatibilité maximal est de 0,85 .

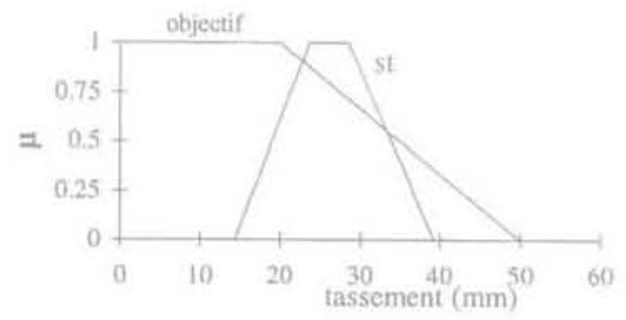

FG.6 Intersection de I'évaluation (st) et de I'objectif (B).

$\mathrm{S}_{1}$ Assessment and $\mathrm{B}$ objective intersection.

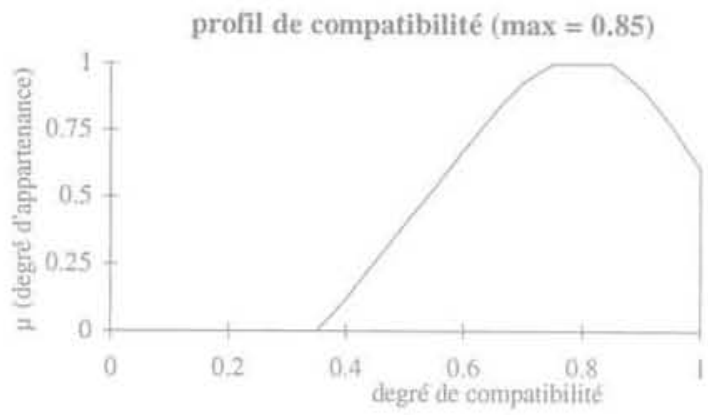

FIG.7 Profil de comptabilité entre l'évaluation et Yobjectif.

Profile of compatibility between objective and assessment.

\section{6}

\section{Exemple 3: \\ Qualités des caractéristiques de sol dans une base de données}

\section{1}

\section{Problème}

Nous disposons d'une base de données qui contient, pour chaque type de matériau, un intervalle de variation pour une caractéristique donnée ; à cha- cun de ces intervalles est associé un indicateur de la qualité de l'estimation de cet intervalle normé entre 0 et 1 (noté conf. : confiance accordée à l'estimation). Un utilisateur fournit une caractéristique d'un sol ainsi que la confiance qu'íl accorde à la mesure : à quelle classe peut-on associer ce matériau?

\section{2}

Exemple (Boissier D., Boulemia C., Semman I., 1998)

Les données fournies par l'utilisateur concernant le module pressiométrique pour une couche d'argile sont: $E=4,5 \mathrm{MPa}$ et conf $E=0,3$. Les cas de référence trouvés dans la bibliographie ou dans une base de données concernant le module pressiométrique pour une couche d'argile sont présentés dans le tableau suivant: Emin et Emax sont les bornes des intervalles estimés (Tableau VII).

TABLEAU VII Domaine de variation de E pour une argile (Nuyens, 73), (Fascicule, 93).

Range of E for a clay (Nuyens, 73), (Fascicule, 93.

\begin{tabular}{l|c|c|c}
\hline Caracteristique & $\begin{array}{c}\text { Nature- } \\
\text { couche }\end{array}$ & $\begin{array}{c}\text { Consistance } \\
\text { _couche }\end{array}$ & $\begin{array}{c}E_{\text {min' }} \\
E_{\text {mine }}\end{array}$ \\
\cline { 3 - 4 } & Argile & Plastique & {$[3,8] \mathrm{Mpa}$} \\
\hline $\mathrm{E}$ & & Raide & {$[8,40] \mathrm{Mpa}$} \\
\hline
\end{tabular}

À chaque intervalle, on associe une modélisation par SEF donnée dans le tableau VIII avec les notations de la figure 1a où U est l'ensemble des cohésions possibles pour les sols.

TABLEAU VIII Résultats de la modélisation des domaines de variation.

Fuzzy parameters.

\begin{tabular}{l|r|r|r|r}
\hline Consistance-couche & $\mathrm{m}-\alpha$ & $\mathrm{m}$ & $\mathrm{n}$ & $\mathrm{n}+\boldsymbol{\beta}$ \\
\hline Molle & 0,00 & 0,00 & 1,50 & 4,50 \\
\hline Plastique & 1,33 & 4,67 & 4,95 & 9,67 \\
\hline Raide & 12,67 & 18,67 & 29,33 & 50,67 \\
\hline
\end{tabular}

Nous constatons sur la figure 8 que le cas décrit par l'utilisateur chevauche les trois cas de référence.

Le calcul de la confiance accordée donne les résultats suivants (tableau IX). 


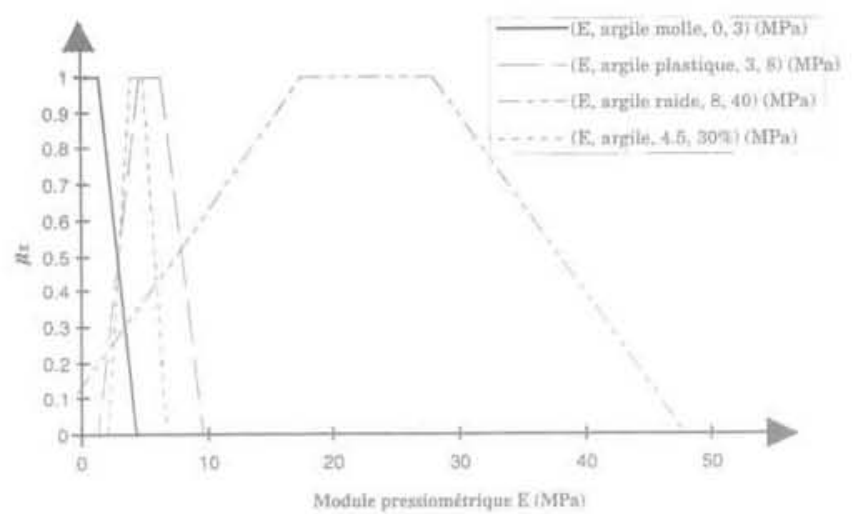

FIG. B Comparaison entre le cas décrit et les cas de référence.

Objective and assessment comparison.

TABLEAUTX Résultats de la discrimination et décision. Results of the discrimination and decision making.

\begin{tabular}{|c|c|c|c|c|}
\hline \multirow[t]{2}{*}{ Caractéristique } & $\begin{array}{l}\text { Nature } \\
\text { _couche }\end{array}$ & $\begin{array}{c}\text { Consistance } \\
\text { _couche }\end{array}$ & $\mathrm{IE}_{\text {mint }}$ & Contiance \\
\hline & & Molle & $\begin{array}{l}{[0,3]} \\
\mathrm{Mpa}\end{array}$ & 0.20 \\
\hline \multirow[t]{2}{*}{$E$} & Argile & Plastique & $\begin{array}{l}{[3,8]} \\
M p a\end{array}$ & 0,95 \\
\hline & & Raide & $\begin{array}{l}{[8,40]} \\
\text { Mpa }\end{array}$ & 0,48 \\
\hline
\end{tabular}

La proposition « la couche considérée est une argile plastique » est retenue, car elle a le degré de vraisemblance le plus élevé et proche de 1 (confiance $=0,95$ ) est retenue : la proposition « la couche considérée est une argile raide $x$ est possible (confiance $=0,48$ ) ; la proposition " la couche considérée est une argile molle \# est rejetée (confiance $=0,2)$.

\section{3}

\section{Conclusion de l'exemple}

La méthode que nous avons proposée permet de quantifier la possibilité qu'une caractéristique appartienne à une classe donnée ; l'exemple très simple présenté ici peut être étendu à des combinaisons d'informations successives permettant chaque fois d'infirmer ou de confirmer cette appartenance.

\section{7}

\section{Exemple 4 : fusion de données}

Nous illustrons la règle de fusion de Dempster pour la question « quelle est la cohésion du sol $n$; nous proposons deux exemples simples sous condition de concordance des données (Fig. 9) et sous condition de conflit (Fig. 10)

Considérons pour un sol donné $\mathrm{c}=80 \mathrm{kPa}$ comme borne supérieure de l'ensemble « ignorance ») (la valeur maximale des cohésions). Supposons que nous ayons obtenu deux preuves (réponses) à cette question.

\section{Cas de preuves concordantes}

Preuve 1: l'expert annonce une cohésion entre 20 et $50 \mathrm{kPa}$; la masse de croyance associée à cette information est de 0,6 .

Preuve 2: un deuxième expert annonce une cohésion entre 40 et $80 \mathrm{kPa}$; la masse de croyance associée à cette information est de 0,7 .

La preuve consensus a donc une masse maximale pour l'intervalle [40, 50] kPa (Fig. 9).

\section{2}

\section{Cas de preuves discordantes}

Preuve 1 : l'expert annonce une cohésion entre 20 et $40 \mathrm{kPa}$; la masse de croyance associée à cette information est de 0,6 .

Preuve 2: un deuxième expert annonce une cohésion entre 50 et $80 \mathrm{kPa}$; la masse de croyance associée à cette information est de 0,7 .

Le principe de normalisation apparait clairement dans cet exemple (Fig, 10). La preuve consensus a donc une masse maximale pour l'intervalle $[50,80] \mathrm{kPa}$; la masse d'ignorance $(0,21)$ est plus importante que dans le cas $1(0,12)$.

\section{3}

\section{Commentaires}

\section{Sur les données}

Les valeurs peuvent provenir de sources diverses que nous avons regroupées sous le vocable « dire d'expert $n$; en particulier elles peuvent être le résultat d'un essai ou d'un calcul.

Les masses de croyance sont de type déclaratif et estimées soit subjectivement soit à l'aide d'une grille d'analyse construite à partir de critères tels que la fiabilité de la méthode d'évaluation, les références de l'organisme donnant l'information, la ressemblance entre les conditions d'obtention de la donnée et les conditions du cas réel (labo/in situ),

\section{7.raba \\ Sur la méthode}

Nous avons appliqué la méthode au cas de deux preuves; elle est bien sûr généralisable au cas de plusieurs preuves : son application impose à l'utilisateur de s'interroger non seulement sur l'information mais aussi sur sa fiabilité, car il est obligé de déclarer la confiance (ou la méfiance) qu'il a en une information.

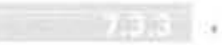 \\ Sur les résultats}

Les masses de croyance obtenues peuvent être utilisées directement en aide à la décision (affectation d'une 


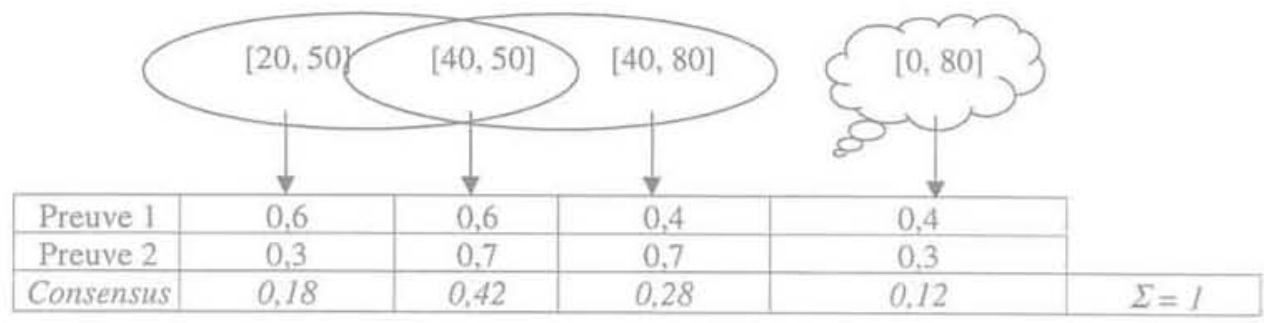

FG.9 Affectation des masses. Cas des preuves concordantes.

Masses allocation. Heterogeneous evidences.

\begin{tabular}{|c|c|c|c|c|}
\hline & $\downarrow$ & $\downarrow$ & $\downarrow$ & \\
\hline Preuve 1 & 0.6 & 0,4 & 0.4 & \\
\hline Preuve 2 & 0,3 & 0,7 & 0,3 & \\
\hline Consensus & 0,18 & 0,28 & 0,12 & $\Sigma=0,58$ \\
\hline Normalis. & 0,31 & 0.48 & 0,21 & $\Sigma=1$ \\
\hline
\end{tabular}

FiG. 10 Affectation des masses. Cas des preuves conflictuelles,

Masses allocation. Conflicting evidences.

valeur à une classe) ou introduites dans un calcul. Elles n'ont qu'une valeur comparative.

\section{8}

\section{Conclusion}

\section{- Intérêts et limites}

L'intérêt principal de ces méthodes possibilistes est d'obliger l'utilisateur à expliciter la confiance qu'il accorde à une information; on ne peut plus faire comme si les données étaient exactes et on accepte de traiter des informations approximatives ; cet intérêt est aussi un des facteurs de rejet de ce type de méthode jugée parfois dérangeante.

Techniquement, ces méthodes peuvent utiliser aussi bien des informations qualitatives que quanti- tatives, des statistiques pauvres ou des champs d'expérimentation complets et les utilitaires d'aide à la modélisation (transformation en SEF par exemple) ne demandent pas de connaissances très sophistiquées.

\section{- SEF ou modèles probabilistes ?}

Les modélisations probabilistes et possibilistes ne sont pas conflictuelles mais alternatives ou complémentaires (La définition fréquentielle de la possibilité permet éventuellement de faire le lien entre les deux distributions). La modélisation possibiliste paraît mieux adaptée à la gestion du qualitatif et de l'information incomplète; elle correspond aux phases primaires de conception et à l'aide à la prise des décisions principales (choix de parti). La modélisation probabiliste demande de l'information quantitative et complète ; elle est mieux adaptée à la phase de calcul détaillé.

\section{Bibliographie}

Aggaggarwal N., Quarrey P., Berry C (1993) - $\alpha$ Application des ensembles flous et de l'aide à la cécision au domaine de forientation professionnelle v. Colloque « Les Applications des ensembles flous o, Nimes، 09/1993, Editions EC2, p. 41-50

Al-Hajiar J., Boissier D., Boulemia C. (1991) - « Building substructural design as fuzzy decision model n. Mexico, CR de 1CASP6, vol. 2 . p. 1076-1083.

Boissier D.. Al-Haijar J. (1990) - « Logique floue et aide à la décision : application a l'infrastructure des bâtiments $₫$. Première table ronde sur la modélisation des processus de conception créative. Cabourg.
Boissier D., Al-Hajjar 1.. Mammeri H. (1995) - «Fiabilité et possibilité en mécanique des sols : application à l'infrastructure des bâtiments 1 . $12 *$ Congrès de mécanique. Strasbourg, 4-8 sept.

Boissier D. Boulemia C. Semaan L. (1998) - "Traitement de lincertain dans une base de connaissances sur le sol o. Première Conférence internationale sur les nouvelles technologies de l'information pour l'aide à la décision dans le domaine du génie civil, Montreal, Canada. 11 . 13 octobre 1998. Miresco E.T., éd., p. $745-756$.

Bouchon-Meunier B. (1995) - La logique floue et ses applications. Amsterdam. Addison-Wesley, p. 84-92.
Dubois D., Prade H. (1983) - « Ranking fuzzy numbers in the sitting of possibility theory $x$. Information Sciences, $n^{\circ} 30$, New York, Elsevier Science Publishing Co., Inc., p. 183-224.

Haofer A.M., Lind N.C. (1974) - « Exact and second invariant second moment code format w. Engineering Mechanics DiviSion ASCE, 100

Lair J. - « Evaluation de la durabilité de systèmes constructifs du bàtiment $n$. Thèse de doctorat de l'unjversité Blaise Pascal, septembre 2000

Shafer G. (1976) - A Mathematical Theory of Evidence. Chichester. Princeton University Press, $297 \mathrm{p}$.

Zadeh L.A. (1965) - «. Fuzzy sets ». Information and Control, $n^{\circ} 8$, p. $338-353$. 